\title{
Decreased outlet angle of the superior cerebellar artery as indicator for dolichoectasia in late onset Pompe disease
}

\author{
Ole Hensel ${ }^{1 *}$, Ilka Schneider ${ }^{1 *}$ (D, Mathias Wieprecht ${ }^{2}$, Torsten Kraya ${ }^{1}$ and Stephan Zierz ${ }^{1}$
}

\begin{abstract}
Background: Lysosomal a-glucosidase deficiency (Pompe disease) not only leads to glycogen accumulation in skeletal muscle, but also in the cerebral arteries. Dolichoectasia of the basilar artery (BA) has been frequently reported. Therefore progression of BA dolichoectasia in late onset Pompe patients (LOPD) was studied.

Methods: BA length, diameter and volume, and cerebral lesions were analysed by MRI/TOF-MR angiography or CT/CT angiography in 20 LOPD patients and 40 controls matching in age, sex- and cardiovascular risk factors. The height of BA bifurcation was assessed semi-quantitatively using the Smoker's criteria and quantitatively by measuring the outlet angle of the superior cerebellar artery (SUCA). Nine patients were followed over 5 years.

Results: The height of the BA bifurcation was abnormal in 12/20 (60\%) LOPD patients and in 12/40 (30\%) matched controls. The SUCA outlet angle was reduced in LOPD patients compared to controls ( $127 \pm 33^{\circ} \mathrm{vs} .156 \pm 32^{\circ}, p=0$. 0024). The diameter, length and volume of the BA were significantly increased in LOPD patients compared to controls. 12/20 (60\%) LOPD patients and 27/40 (68\%) controls presented white matter lesions. During 5 years 2/9 LOPD patients developed an abnormal height of BA bifurcation according to the Smoker's criteria and in all patients the SUCA outlet angle decreased $\left(138 \pm 34^{\circ}\right.$ vs. $\left.128 \pm 32^{\circ}, p=0.019\right)$. One patient with prominent basilar dolichoectasia experienced a thalamic hemorrhage.

Conclusion: Pompe disease is associated with BA dilation, elongation and elevated bifurcation height of the BA which might result in cerebrovascular complications. The SUCA outlet angle seems to be useful for monitoring the progression of BA dolichoectasia.
\end{abstract}

Keywords: Late onset Pompe disease (LOPD), Dolichoectasia of basilar artery, Dilative arteriopathy, Height of basilar bifurcation, Glycogenosis type II, Superior cerebellar artery (SUCA, SCA), Acid maltase deficiency

\section{Background}

Pompe disease (glycogenosis type II, OMIM\# 232300) is a multi-systemic disorder caused by reduced activity of the lysosomal acid glycosidase $\alpha$ presenting with predominant muscular weakness, respiratory and cardiac insufficiency [1]. Autopsy studies showed glycogen accumulation also in smooth muscles of the tunica media of cerebral arteries indicating involvement of the cerebrovascular system [2]. Microangiopathies (e.g. white matter lesions, microbleeds) and macroangiopathies (e.g.

\footnotetext{
* Correspondence: ole.hensel@medizin.uni-halle.de; ilka.schneider@uk-halle.de ${ }^{1}$ Department of Neurology, University hospital Halle/Saale, Ernst-Grube-Str. 40, 06120 Halle/Saale, Germany

Full list of author information is available at the end of the article
}

dolichoectasia and aneurysms mostly of vertebrobasilar arteries) have been repeatedly reported in late onset Pompe disease (LOPD) [3]. Cerebrovascular complications might be crucial for long term survival of LOPD patients as these cerebrovascular changes were associated with intracranial haemorrhages, compression of cranial nerves and brainstem, and stroke [3-6]. Recent studies have demonstrated that dolichoectasia of the basilar artery (BA) is the most striking cerebrovascular finding in LOPD patients [3]. A preliminary study showed a significant dilation of vertebrobasilar arteries compared to controls [7]. However, little is known about the progression of this arterial remodelling in the course of LOPD. Therefore this study investigated how BA 
characteristics of 20 LOPD patients differ from those of 40 subjects that individually matched the LOPD patients in age-, sex-, and risk factors. Secondly the progression of BA arteriopathy during a 5 year observation period was analysed retrospectively for 9 of the 20 LOPD patients. Finally the occurrence of cerebral lesions was examined.

\section{Patients and controls}

Twenty LOPD patients with genetically and biochemically confirmed diagnosis of Pompe disease (age $53.7 \pm$ 14.6 years, range 19 to 81 ) were studied. All Patients were on enzyme replacement therapy (ERT) (mean 68.8 \pm 42.8 months; range 9 to 135 months). Table 1 summarizes clinical and molecular data of LOPD patients. Follow up MRI examination was performed after 5 years in 9/20 LOPD patients.

For each Pompe patient two controls $(n=40$; age 54.0 \pm 14.7 years, range 19 to 80 ) were recruited that matched in gender, age (maximal \pm 3 years) and number of cerebrovascular risk factors (maximal \pm 1 ). Relevant risk factors were defined as hypertension, hyperlipidemia, obesity, smoking and diabetes. The control group consisted of patients with other neurological diseases that underwent cerebral MRI imaging for diagnostic purpose. This included headache (11/40), TIA and minor cardioembolic stroke (7/10), peripheral neuropathies (5/ $40)$, seizures $(4 / 40)$, myopathies other than Pompe disease $(3 / 40)$, other diagnoses $(10 / 40)$ were retrobulbar optic neuritis, anterior ischemic optic neuropathy, spinocerebellar syndrome, dementia, and psychiatric diseases.

\section{Methods}

All participants except one LOPD patient underwent cerebral TOF-MR angiography and FLAIR-MRI. Additional T2*-weighted sequences were performed in 19 LOPD patients (1.5 Tesla Magnetom Sonata Vision, Siemens, Erlangen, Germany). Because of cardiac pacemaker one LOPD patient was examined by cerebral $\mathrm{CT}$ and $\mathrm{CT}$ angiography. Follow up imaging was performed with the identical MRI scanner and MRI sequences. There were slight differences in the TOF-MRI protocol. All images were anonymized and reviewed by the neuroradiologist (M.W.) and by a skilled neurologist (O.H.). Smoker's criteria for basilar dolichoectasia [8] were applied for characterisation of the BA: The semi-quantitative grading of the height of the BA bifurcation was assessed (grade 0: at or below dorsum sellae; grade 1: within suprasellar cistern; grade 2: at

Table 1 Clinical and molecular data of 20 LOPD patients

\begin{tabular}{|c|c|c|c|c|c|c|c|}
\hline Pat.-Nr. & Sex & GAA-Genotype & & Age [years] & $\begin{array}{l}\text { Duration disease } \\
\text { (years) }\end{array}$ & $\begin{array}{l}\text { Duration ERT } \\
\text { (month) }\end{array}$ & $\begin{array}{l}\text { Cardio-vascular risk } \\
\text { factors }\end{array}$ \\
\hline$\overline{P 1^{\mathrm{a}}}$ & $\mathrm{F}$ & IVS1 $(-13 T>G)$ & IVS9 G > C) & 26 & 15 & 67 & $\mathrm{HL}$ \\
\hline P2 & $\mathrm{F}$ & p.L552P & p.P493L & 37 & 10 & 33 & $\mathrm{HL}, \mathrm{O}$ \\
\hline$P 3^{\mathrm{a}}$ & M & IVS1 $(-13 \mathrm{~T}>\mathrm{G})$ & c.2136-7delGT & 50 & 40 & 116 & HYP, HL \\
\hline$P 4^{\mathrm{a}}$ & M & IVS1 $(-13 \mathrm{~T}>\mathrm{G})$ & p.W499R & 50 & 18 & 130 & $\mathrm{DM}, \mathrm{HL}$ \\
\hline$P 5^{\mathrm{a}}$ & M & IVS1 $(-13 \mathrm{~T}>\mathrm{G})$ & p.P493L & 52 & 15 & 63 & HYP, HL \\
\hline$P 6^{a}$ & $\mathrm{~F}$ & IVS1 $(-13 T>G)$ & p.L552P & 53 & 17 & 99 & HYP, HL, O, S \\
\hline$P 7^{\mathrm{a}}$ & M & IVS1 $(-13 \mathrm{~T}>\mathrm{G})$ & p.C103G & 59 & 14 & 135 & HYP, DM, HL, O \\
\hline$P 8^{a}$ & $\mathrm{~F}$ & IVS1 $(-13 \mathrm{~T}>\mathrm{G})$ & p.P493L & 61 & 21 & 63 & HYP, HL \\
\hline P9 & M & IVS1 (-13 T> G) & p.G309R & 64 & 11 & 14 & HYP, DM, O \\
\hline $\mathrm{P} 10^{\mathrm{a}}$ & $\mathrm{F}$ & IVS1 $(-13 \mathrm{~T}>\mathrm{G})$ & del exon 18 & 75 & 40 & 103 & HYP \\
\hline P11 & M & IVS1 (-13 T> G) & c.832delC & 45 & 6 & 17 & HYP, HL \\
\hline P12 & $\mathrm{F}$ & IVS1 (-13 T > G) & c.525delT & 66 & 7 & 33 & $\mathrm{HYP}, \mathrm{HL}$ \\
\hline P13 & M & IVS1 (-13 T > G) & IVS1 $(-13 \mathrm{~T}>\mathrm{G})$ & 61 & 13 & 29 & HYP, HL \\
\hline P14 & $\mathrm{F}$ & IVS1 (-13 T > G) & c. $2481+102 \_2646+31 \mathrm{del}$ & 54 & 18 & 44 & $\mathrm{HYP}, \mathrm{HL}$ \\
\hline P15 & $\mathrm{F}$ & IVS1 (-13 T > G) & c. $2481+102 \_2646+31$ del & 56 & 19 & 48 & HYP, HL \\
\hline P16 ${ }^{\mathrm{a}}$ & $\mathrm{F}$ & IVS1 $(-13 \mathrm{~T}>\mathrm{G})$ & $\mathrm{c} 307 \mathrm{~T}>\mathrm{G}$ & 49 & 23 & 119 & $\mathrm{HL}$ \\
\hline P17 & M & IVS1 (-13 T > G) & p.G309R & 81 & 6 & 37 & HYP, HL \\
\hline P18 & M & IVS1 (-13 T > G) & c.794delG & 49 & 13 & 84 & NONE \\
\hline P19 & $\mathrm{F}$ & IVS1 (-13 T> G) & c. $925 G>A$ & 61 & 2 & 9 & HYP \\
\hline P20 & M & IVS1 (-13 T> G) & c.525delT & 19 & 19 & 132 & NONE \\
\hline
\end{tabular}

Patient P1-P10 were numbered according to their number in the previous study by Hensel et al. [7]. P5 + P8 and P14 + 15 were siblings; a patients with 5 years follow-up; Abbr.: ERT enzyme replacement therapy, GAA Glucosidase alpha gene. Risk factors: DM diabetes mellitus, $H L$ hyperlipidemia, $H Y P$ Hypertension, $O$ obesity, S smoking 
level of third ventricle; grade 3: indenting and elevating floor of third ventricle; abnormal $\geq$ grade 2) and the lateral displacement of the BA (grade 0: midline throughout; grade 1: medial to lateral margin of clivus or dorsum sellae; grade 2: lateral to lateral margin of clivus or dorsum sellae; grade 3: in cerebellopontine angle cistern; abnormal $\geq$ grade 2). The inner diameter of BA was quantified proximally (1 mm after BA origin), at the middle (half of the anatomic BA length), and distally (3 $\mathrm{mm}$ before $\mathrm{BA}$ bifurcation). The smallest diameter on axial TOF-MR images was used. For calculation of BA volume the smallest diameter was measured in $1 \mathrm{~mm}$ slices. For all diameter measurements, an intensity-based analysis was used (FWHM Line-plugin of ImageJ-Software, version $1.51 \mathrm{~h}$, $\mathrm{NIH}$, USA). This allowed a precise and user independent diameter measurement [9]. The anatomical and the linear length of the BA were measured from the conjunction of both vertebral arteries (VA) to the BA bifurcation (as depicted in Figure 1a). Additionally the outlet angle of the superior cerebellar artery (SUCA) from the BA was assessed using hypotenuse (SUCA) and adjacent side (BA) at $5 \mathrm{~mm}$ distance (as depicted in Figure 1). The SUCA outlet angle was defined as the sum of the left and the right outlet angle. This method was chosen to take into account that the SUCA outlet might differ between left and right side because of a frequent asymmetry of the SUCA. In FLAIR-MRI, the deep white matter lesions were graded according to the Fazekas score [10]. The gradient-echo T2weighted $(\mathrm{T} 2 *)$ images were screened for cerebral microbleeds. Statistical analysis was conducted using SigmaStat (SYSTAT Software GmbH, version 4.0, Germany). A $p$ value $<0.05$ was considered significant. The research protocol was approved by the Ethical Committee of the medical faculty of the Martin-Luther-University HalleWittenberg.

\section{Results}

BA characteristics in 20 LOPD patients and $\mathbf{4 0}$ controls

The height of the BA bifurcation was abnormal in 12/20 LOPD patients $(60 \%)$ and in $12 / 40$ matched controls (30\%). Abnormal lateral displacement of the BA was observed in $1 / 20$ LOPD patients (5\%) and in 1/40 matched controls (2.5\%). LOPD patients had significantly dilated middle and distal BA diameters compared to matched controls. The anatomic length, linear length, and volume of the BA in LOPD patients were also significantly higher than in the control group. The SUCA outlet angles were significantly reduced in LOPD patients compared to controls (Table 2).

In LOPD patients, there was a positive correlation between BA anatomical length (Pearson $r=0.626, p=0.0$. 003), linear length (Pearson $r=0.522, p=0.018$ ), distal diameter (patients: Pearson $r=0.536, p=0.015$ ), and volume (Pearson $r=0.516, p=0.020$ ) with age.

The SUCA outlet angle (Figure 2) was negatively correlating with age in LOPD patients (Pearson $r=-0.605, p=$ 0.006 ) and in controls (Pearson $r=-0.483, p=0.002$ ).

In total, in the cohort of all subjects (LOPD patients and controls) the SUCA outlet angle correlated with BA anatomic length (Pearson $r=-0,558, p<0.001$ ), the BA linear length (Pearson $r=-0.546, p<0.001$ ), the BA volume (Pearson $r=-0,545, p<0.001$ ). Correlations according to the Smoker criteria were as follows: with BA diameter (distal: Pearson $r=-0.407, p=0.001$; middle: Pearson $r=-0.363, p=0.005$; proximal: Pearson $r=$ $-0.280, p=0.032$ ), and the grade of the BA bifurcation

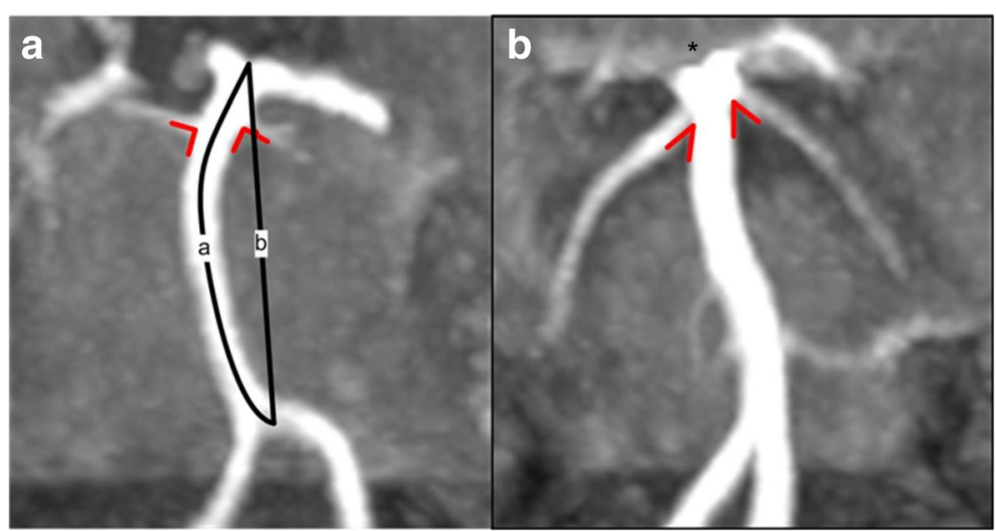

Fig. 1 Basilar artery (BA) and the outlet angle of the superior cerebellar artery (SUCA) Assessment of the BA length and bifurcation height and SUCA outlet angle. a) A 57 year old male control patient with normal height of the BA bifurcation (grade 0 according to Smoker criteria [8]) and nearly perpendicular SUCA outlet from the basilar artery (angle right: $85^{\circ}$, angle left: $75^{\circ}$; SUCA outlet angle of $160^{\circ}$ as defined by sum of left and right side). b) A 61 year old female LOPD patient with dilated basilar artery, cranial shifted BA bifurcation indenting the third ventricle and hypothalamus ${ }^{*}$, grade 3 according to Smoker's criteria [8]) and low SUCA outlet angle (angle right: $48^{\circ}$, angle left: $31^{\circ}$; SUCA outlet angle of $79^{\circ}$ as defined by sum of left and right side). Schematic drawings indicate measurements of SUCA outlet angles (red angles) and measurements of basilar artery: anatomic length (line a) and linear length (line b) 
Table 2 Characteristics of basilar artery in LOPD and controls measured by TOF-MRA LOPD $(n=20)$ controls $(n=40) \quad p$-value $\neq$

Basilar artery characteristics

- Diameter (mm)

\begin{tabular}{|c|c|c|c|}
\hline $\begin{array}{l}\text { - proximal } \\
\text { - middle } \\
\text { - distal }\end{array}$ & $\begin{array}{l}3.5 \pm 0.8 \\
3.2 \pm 1.2 \\
3.2 \pm 1.1\end{array}$ & $\begin{array}{l}3.1 \pm 1.0 \\
2.4 \pm 0.4 \\
2.7 \pm 0.6\end{array}$ & $\begin{array}{l}p=0.130 \\
p=0.0006 \\
p=0.013\end{array}$ \\
\hline - Anatomical length (mm) & $34.0 \pm 6.6$ & $30.7 \pm 5.2$ & $p=0.039$ \\
\hline - Linear length (mm) & $31.3 \pm 3.7$ & $29.1 \pm 4.0$ & $p=0.051$ \\
\hline - Volume (ml) & $0.209 \pm 0.164$ & $0.113 \pm 0.547$ & $p=0.0014$ \\
\hline \multicolumn{4}{|l|}{ Smoker criteria [8] } \\
\hline $\begin{array}{l}\text { - Abnormal lateral BA } \\
\text { displacement ( } \geq \text { grade 2) }\end{array}$ & $1 / 20(5 \%)$ & $1 / 40(2.5 \%)$ & $p=1.00$ \\
\hline $\begin{array}{l}\text { - Abnormal height of BA } \\
\text { bifurcation ( } \geq \text { grade } 2 \text { ) }\end{array}$ & $12 / 20(60 \%)$ & $12 / 40(30 \%)$ & $p=0.049$ \\
\hline \multicolumn{4}{|l|}{ Other parameter } \\
\hline SUCA outlet angle $\left({ }^{\circ}\right)$ & $127 \pm 33^{\mathrm{a}}$ & $156 \pm 32$ & $p=0.0024$ \\
\hline White matter lesions & $12 / 19(63 \%)^{b}$ & $27 / 40(68 \%)$ & $p=0.77$ \\
\hline
\end{tabular}

¥ application of two sample t-test; ${ }^{a}$ in one LOPD patient the BA bifurcation was such abnormal, that SUCA outlet angle was not measurable; ${ }^{b}$ one LOPD patient had a CT

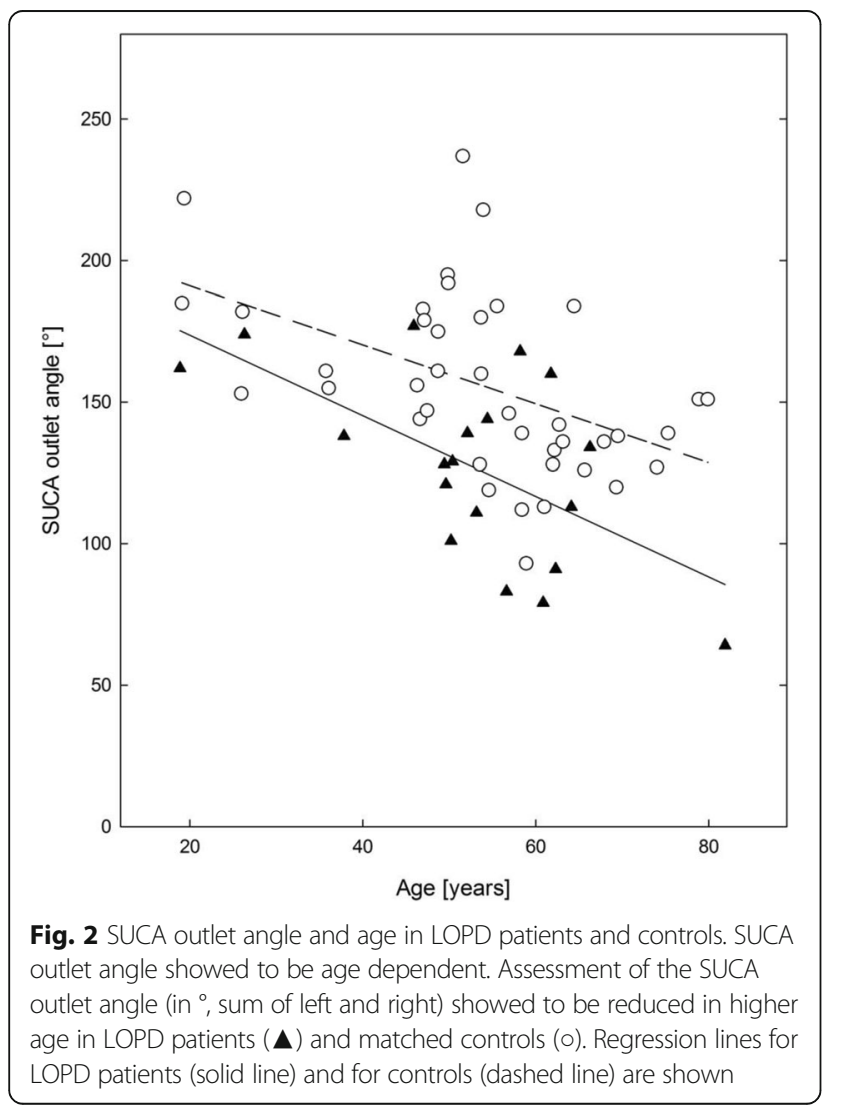

height (Pearson $r=-0.653, p<0.001$ ) and with the extend of the lateral displacement (Pearson $r=-0.287, p=$ $0.028)$.

There was no gender difference in BA diameter, anatomic length, linear length, volume or SUCA outlet angle in LOPD patients or controls.

\section{BA characteristics during 5 year follow-up in 9 LOPD patients}

At baseline three patients (P8, P10, P16) had an abnormal height of the BA bifurcation. After 5 years two more patients (P3, P6) presented an abnormal BA height. In all 9 patients the individual SUCA outlet angle showed to be decreased after the observation period. On a group level this decrease was significant (Table 3). However, no significant changes were seen for BA diameter, anatomic length, linear length and volume.

\section{Other cerebral abnormalities}

During the observation period of 5 years, there were no major cerebrovascular events in 8/9 LOPD patients. However, one of the patients, a 75 year- old female, experienced an intracranial thalamic haemorrhage on the left side that led to complete immobilisation and subsequent death. This patient presented a remarkable dolichoectasia of the BA with an extreme cranial and posteriorly shifting of the BA bifurcation (Additional file 1 Figure S1).

White matter lesions were observed in 12/19 LOPD patients $(63 \%)$ and $27 / 40$ controls (68\%). According to the

Table 3 Characteristics of basilar artery in 9 LOPD patients during 5 year follow up measured by TOF-MRA

\begin{tabular}{|c|c|c|c|}
\hline & Baseline & $\begin{array}{l}5 \text { year } \\
\text { follow-up }\end{array}$ & $p$ values \\
\hline \multicolumn{4}{|l|}{ Basilar artery characteristics } \\
\hline \multicolumn{4}{|l|}{ - Diameter (mm) } \\
\hline $\begin{array}{l}\text { - proximal } \\
\text { - middle } \\
\text { - distal }\end{array}$ & $\begin{array}{l}3.8 \pm 1.8 \\
3.2 \pm 0.7 \\
2.9 \pm 0.8\end{array}$ & $\begin{array}{l}3.6 \pm 0.9 \\
3.5 \pm 1.5 \\
3.3 \pm 1.4\end{array}$ & $\begin{array}{l}p=0.512 \\
p=0.584 \\
p=0.143\end{array}$ \\
\hline - Anatomic length (mm) & $33.2 \pm 7.5$ & $33.4 \pm 8.2$ & $p=0.808$ \\
\hline - Linear length (mm) & $30.3 \pm 2.9$ & $30.1 \pm 3.9$ & $p=0.799$ \\
\hline - Volume (ml) & $0.214 \pm 0.138$ & $0.240 \pm 0.211$ & $p=0.475$ \\
\hline \multicolumn{4}{|l|}{ Smokers criteria } \\
\hline $\begin{array}{l}\text { - Abnormal lateral } \\
\text { BA displacement }\end{array}$ & $0 / 9(0 \%)$ & 0/9 (0\%) & $p=1.00$ \\
\hline $\begin{array}{l}\text { - Abnormal height of } \\
\text { BA bifurcation }\end{array}$ & $3 / 9(33 \%)$ & $5 / 9(56 \%)$ & $p=0.64$ \\
\hline \multicolumn{4}{|l|}{ Other parameter } \\
\hline SUCA outlet angle $\left(^{\circ}\right)$ & $138 \pm 34$ & $128 \pm 32$ & $p=0.019$ \\
\hline White matter lesions ${ }^{\mathrm{b}}$ & $5 / 8(63 \%)$ & $5 / 8(63 \%)$ & $p=1.00$ \\
\hline
\end{tabular}

aTOF-MRA was done with the same MR scanner, but different TOF-MR protocols; bone LOPD patient had a CT scan 
Fazekas score grading of the LOPD patients was as follows: $40 \%$ grade $0,30 \%$ grade $1,25 \%$ grade $2,5 \%$ grade 3 . Grading of controls was: $32.5 \%$ grade $0,40 \%$ grade $1,20 \%$ grade $2,7.5 \%$ grade 3 . On a group level, Fazekas grade was not different in the LOPD patients compared to controls $(0.95 \pm 0.95$ versus $1.05 \pm 0.96)$. In controls, there were no major cerebrovascular abnormalities (e.g. tumor, major stroke, aneurysms).

None of the 9 LOPD patients showed progression of deep white matter lesions in within the 5 year period according to the Fazekas criteria.

The lowest SUCA outlet angle was found in the oldest LOPD patient (P17: 64'), who had a dilated BA and an additional severe mid-basilar stenosis. Other low SUCA outlet angles were found in one patient (P8: $79^{\circ}$ ) with microbleeds that were exclusively present in the posterior circulation and in one (P14: $83^{\circ}$ ) with a bilaterally fetal-type circle of Willis.

\section{Discussion}

\section{Dolichoectasia in patients and controls}

The present study on 20 LOPD patients under enzyme replacement therapy (ERT) showed a significant dilation of the BA (regarding diameter and lengths) compared to matched controls. Similar changes have previously reported in 10 of these 20 patients [7]. However, in the present study a more accurate and objective assessment of the vessel diameter was performed using FWHM, based on the pixel-wise intensity [9]. The findings in our cohort are consistent with those of previous studies that found predominant dolichoectasia in the posterior cerebral circulation in LOPD patients with and without ERT $[3,11]$. The reason for these changes remains unclear. It was speculated that glycogen deposition in the smooth muscles of the intima of arterial vessels provokes their dilation [2, 12]. In this study all patients received ERT and its impact on cerebral vessels remains enigmatic. However, there is a report that suggests glycogen clearance in smooth muscle via ERT in the arrector pili muscle [13].

However, vertebrobasilar dolichoectasia is not a specific phenomenon in Pompe disease: In general the posterior circulation has less sympathetic innervation than the anterior circulation leading to a predisposition to deformity, when exposed to increased blood flow and pressure [14]. Subsequently, there is physiological progressive luminal dilation, fragmentation of the internal elastic lamina, and elastin loss in aging individuals, that mainly affect the vertebrobasilar arteries [15-17]. Besides a higher age, also a male gender and cardiovascular risk factors (hypertension, smoking, obesity, diabetes, or dyslipidemia) have been found to be associated with evolution of dolichoectasia [18]. Therefore LOPD patients were individually matched with controls of equal epidemiological and cardiovascular parameters.

Since signs of dolichoectasia were observed in both groups, the LOPD patients and the matched controls, additional factors for the development of dilative arteriopathy have to be assumed that result from the comorbidities. However, the BA ectasia was more pronounced in the LOPD cohort compared to controls. It is therefore suggested that Pompe disease acts as independent factor for the development of BA dolichoectasia.

Until now, the most validated parameters for BA evaluation are the semi-quantitative Smoker's criteria [8]: An abnormal height of BA bifurcation implies that the BA bifurcation is cranial shifted to the level of third ventricle floor or higher. Normally, the SUCA originates shortly under the BA bifurcation at the height of the free edge of the tentorium cerebelli [19] and the SUCA outlet angle is nearly $180^{\circ}$. If the SUCA origin and the adjacent basilar bifurcation shift cranial, then the SUCA outlet angle will consequently decrease. The SUCA is most consistent and the angle is easily obtained from coronal CT- or MR angiography images, independently from different imaging protocols. Therefor in this study the outlet angle of the SUCA was introduced as a novel parameter for a quantitative analysis of this cranial shift of BA bifurcation. It was demonstrated that the SUCA outlet angle in LOPD patients was significantly reduced compared to controls together with other indicators of a prominent BA ectasia in these patients. Additionally, there was a significant correlation of the SUCA outlet angle with all parameters of the Smoker's criteria: BA diameter, bifurcation height and lateral displacement.

\section{Analysis of progression of dolichoectasia in LOPD patients}

In the present study, according to the Smoker's criteria [8], only 3/9 LOPD patients showed an abnormal height of the BA bifurcation at baseline but 5/9 LOPD patients after 5 years follow up. In the 5 year follow up analysis there was a significant decline of the SUCA outlet angle in the cohort of 9 LOPD patients on ERT that suggests a progressive dolichoectasia in these patients. The higher slope of the regressionline of the LOPD cohort compared to controls in the age dependent analysis of the SUCA outlet suggests that these differences result from disease specific mechanism rather from the aging of the patients.

However, other parameters that define BA dolichoectasia (length, diameter, and lateral displacement of the BA) remained nearly unchanged. Similar results were reported in a recent study showing stable vertebrobasilar diameters over a period of up to 10 years in 5 LOPD patients [20]. This might be due to the relatively small diameter and lengths of the BA. Changes of these 
parameters may need time to evolve and are therefore not easily detectable in the 5 year observation period. However, presumed that all vertebrobasilar arteries are affected by a weakening of the vessel wall, and then the cranial migration of the BA bifurcation might be a result of a summation effect of the elongation of the BA plus the adjacent vertebral arteries. Since vasodilation results in reduced flow velocity, the dilation of cerebral arteries might be underestimated in TOF-based MRA. Limitation of this study might be the use of slight differently TOF-MRI protocols in the follow up cerebral imaging. However, this has no impact on the SUCA outlet angle. It is suggested that the SUCA outlet angle is a sensitive surrogate marker for BA dolichoectasia, which detects the elevation of the height of basilar bifurcation in a more precise and quantitative way than the Smoker's grading.

\section{Clinical relevance}

It is well known that dolichoectasia can lead to brainstem compression, hydrocephalus microbleeds and stroke [18, $21,22]$. There are several reports on strokes $[6,23]$ and intracranial mirco - and macrohemorrhages $[4,5,24]$ in Pompe patients. In the actual LOPD cohort a fatal thalamic hemorrhage occurred in one patient with the most prominent basilar dolichoectasia. It can be speculated, that the vertebrobasilar pathology might at least has contributed to the hemorrhage in this patient. However, in all 9 patients there was no progression of white matter lesions after the follow up of 5 years.

It seems therefore appropriate, that patients without cerebrovascular abnormalities undergo a follow up of MRI analysis after 5 years. In patients with cerebrovascular abnormalities, we recommend the strict treatment of cerebrovascular risk factors (e.g. hypertension) and follow up imaging after 1 to 2 years.

\section{Conclusion}

Dilation and elongation of the BA is pronounced in LOPD patients under ERT compared to matched controls. This suggests that LOPD acts as an independent risk factor for basilar dolichoectasia. The study demonstrates a decreasing SUCA outlet angle as surrogate marker for a progressive elevation of the BA bifurcation in the disease course of LOPD during a 5 year observation period. The SUCA outlet angle seems to be useful for detection of progression of vertebrobasilar dolichoectasia.

\section{Additional file}

Additional file 1: Figure S1. A case of very prominent vertebrobasilar dolichoectasia. Vertebrobasilar arteries of a 75 year old female LOPD patient. Basilar artery shows to be dilated and elongated with massive cranial and posterior shift of the BA bifurcation height (A coronal, B sagittal view). This
LOPD patient experienced a left side thalamic hemorrhage. As consequence of massive BA shift the SUCA outlet angle was not reasonable measurable. Abbr. 1 distal part of basilar artery, 2 bifurcation of basilar artery, 3 posterior cerebral artery, 4 superior cerebellar artery, 5 anterior inferior cerebellar artery, 6 vertebral artery. (PDF $150 \mathrm{~kb}$ )

\section{Abbreviations}

BA: Basilar artery; ERT: Enzyme replacement therapy; LOPD: Late onset Pompe disease; SUCA: Superior cerebellar artery; VA: Vertebral artery

\section{Acknowledgements}

Tobias Mueller, MD (Department of Neurology, University hospital Martin-LutherUniversity, Halle/Saale, Germany) for supervision of the dupexsonographic studies; Sabine Koesling, Prof. (Department of Diagnostic Radiology, University hospital Martin-Luther-University, Halle/Saale, Germany) for supervision of the radiological analysis; Titus Heyke (VitalAire GmbH, Leipzig, Germany) for technical support of patient CPAP ventilation during MR examination; Torben Schneider, PhD (Philips Healthcare, UK) edited the manuscript for non-intellectual content

\section{Statistical analysis}

Conducted by Ole Hensel, University hospital Halle, Halle/Saale, Germany.

Funding

No funding.

Availability of data and materials

It is stated that the authors have full access to all data and the right to publish any and all data. Please contact the corresponding author for ata requests.

\section{Authors' contributions}

IS, concept and design, acquisition of data, analysis and interpretation of data. $\mathrm{OH}$, study concept and design, acquisition of data, analysis and interpretation of data. MW, acquisition of data, analysis and interpretation of data. TK, acquisition of data, analysis and interpretation of data. SZ, study supervision, analysis and interpretation of data, critical revision of manuscript for intellectual content. All authors read and approved the final manuscript.

Ethics approval and consent to participate

All participants gave their informed consent to take part in the study. The research protocol was approved by the Ethical Committee of the Medical Faculty of the Martin-Luther-University Halle-Wittenberg.

\section{Consent for publication}

All participants gave their informed consent to data publication. All authors have read the manuscript and agreed the submission an publication in the OJRD.

\section{Competing interests}

Ole Hensel - Reports no disclosures; Ilka Schneider - Honoraria for lectures, travel expenses from Genzyme Corporation; Mathias Wieprecht - Reports no disclosures; Torsten Kraya - Honoraria for lectures, travel expenses from Genzyme Corporation; Stephan Zierz - Honoraria for lectures, travel expenses from Genzyme Corporation.

\section{Publisher's Note}

Springer Nature remains neutral with regard to jurisdictional claims in published maps and institutional affiliations.

\section{Author details}

${ }^{1}$ Department of Neurology, University hospital Halle/Saale, Ernst-Grube-Str. 40, 06120 Halle/Saale, Germany. ${ }^{2}$ Department of Diagnostic Radiology, University hospital Halle, Ernst-Grube-Str. 40, Halle/Saale, Germany. 
Received: 30 December 2017 Accepted: 22 March 2018 Published online: 13 April 2018

\section{References}

1. Schneider I, Zierz S. Profile of alglucosidase alfa in the treatment of Pompe disease: safety, efficacy, and patient acceptability. Research and Reports in Endocrine Disorders. 2015;6:1-9.

2. Kretzschmar HA, Wagner H, Hubner G, Danek A, Witt TN, Mehraein P. Aneurysms and vacuolar degeneration of cerebral arteries in late-onset acid maltase deficiency. J Neurol Sci. 1990;98:169-83.

3. Montagnese F, Granata F, Musumeci O, Rodolico C, Mondello S, Barca E, Cucinotta M, Ciranni A, Longo M, Toscano A. Intracranial arterial abnormalities in patients with late onset Pompe disease (LOPD). J Inherit Metab Dis. 2016;2(s1):S48.

4. Zhang B, Zhao Y, Liu J, Li L, Shan J, Zhao D, Yan C. Late-onset Pompe disease with complicated intracranial aneurysm: a Chinese case report. Neuropsychiatr Dis Treat. 2016;12:713-7.

5. Brettschneider J, Sperfeld AD, Ludolph AC, Kassubek J. Intracerebral hemorrhage in a patient with glycogenosis type II (Pompe disease): is there a pathophysiological relationship? Muscle Nerve. 2008;38:1211-2.

6. Huded V, Bohra V, Prajapati J, DeSouza R, Ramankutty R: Stroke in youngdilative Arteriopathy: a clue to late-onset Pompe's disease? J stroke Cerebrovasc dis 2016.

7. Hensel O, Hanisch F, Stock K, Stoevesandt D, Deschauer M, Muller T. Morphology and function of cerebral arteries in adults with pompe disease. JIMD Rep. 2015:20:27-33.

8. Smoker WR, Corbett JJ, Gentry LR, Keyes WD, Price MJ, McKusker S. Highresolution computed tomography of the basilar artery: 2. Vertebrobasilar dolichoectasia: clinical-pathologic correlation and review. AJNR Am J Neuroradiol. 1986;7:61-72.

9. Hoogeveen RM, Bakker CJ, Viergever MA. Limits to the accuracy of vessel diameter measurement in MR angiography. J Magn Reson Imaging. 1998;8: 1228-35.

10. Fazekas F, Chawluk JB, Alavi A, Hurtig HI, Zimmerman RA. MR signal abnormalities at $1.5 \mathrm{~T}$ in Alzheimer's dementia and normal aging. In AJR Am J Roentgenol. 1987;149:351-6.

11. Pichiecchio A, Sacco S, De Filippi P, Caverzasi E, Ravaglia S, Bastianello S, Danesino C. Late-onset Pompe disease: a genetic-radiological correlation on cerebral vascular anomalies. J Neurol. 2017:264(10):2110-18.

12. Hobson-Webb LD, Proia AD, Thurberg BL, Banugaria S, Prater SN, Kishnani PS. Autopsy findings in late-onset Pompe disease: a case report and systematic review of the literature. Mol Genet Metab. 2012;106:462-9.

13. Katona I, Weis J, Hanisch F. Glycogenosome accumulation in the arrector pili muscle in Pompe disease. Orphanet J Rare Dis. 2014;9:17.

14. Samim M, Goldstein A, Schindler J, Johnson MH. Multimodality imaging of Vertebrobasilar Dolichoectasia: clinical presentations and imaging Spectrum. Radiographics. 2016;36:1129-46.

15. Passero SG, Rossi S. Natural history of vertebrobasilar dolichoectasia. Neurology. 2008;70:66-72.

16. Gutierrez J, Honig L, Elkind MS, Mohr JP, Goldman J, Dwork AJ, Morgello S, Marshall RS. Brain arterial aging and its relationship to Alzheimer dementia. Neurology. 2016;86:1507-15.

17. Roth W, Morgello S, Goldman J, Mohr JP, Elkind MS, Marshall RS, Gutierrez J. Histopathological differences between the anterior and posterior brain arteries as a function of aging. Stroke. 2017;48:638-44.

18. Ikeda K, Nakamura Y, Hirayama T, Sekine T, Nagata R, Kano O, Kawabe K, Kiyozuka T, Tamura M, Iwasaki Y. Cardiovascular risk and neuroradiological profiles in asymptomatic vertebrobasilar dolichoectasia. Cerebrovasc Dis. 2010;30:23-8.

19. Hardy DG, Peace DA, Rhoton AL Jr. Microsurgical anatomy of the superior cerebellar artery. Neurosurgery. 1980;6:10-28.

20. Garibaldi M, Sacconi S, Antonini G, Desnuelle C. Long term follow-up of cerebrovascular abnormalities in late onset Pompe disease (LOPD). J Neurol. 2017;264:589-90.

21. Tanaka M, Sakaguchi M, Miwa K, Okazaki S, Furukado S, Yagita Y, Mochizuki H, Kitagawa K. Basilar artery diameter is an independent predictor of incident cardiovascular events. Arterioscler Thromb Vasc Biol. 2013;33:2240-4.

22. Lou M, Caplan LR. Vertebrobasilar dilatative arteriopathy (dolichoectasia). Ann N Y Acad Sci. 2010;1184:121-33.
23. Quenardelle V, Bataillard M, Bazin D, Lannes B, Wolff V, Echaniz-Laguna A. Pompe disease presenting as an isolated generalized dilative arteriopathy with repeated brain and kidney infarcts. J Neurol. 2015;262:473-5.

24. Sandhu D, Rizvi A, Kim J, Reshi R. Diffuse cerebral microhemorrhages in a patient with adult-onset Pompe's disease: a case report. J Vasc Interv Neurol. 2014;7:82-5.

\section{Submit your next manuscript to BioMed Central and we will help you at every step:}

- We accept pre-submission inquiries

- Our selector tool helps you to find the most relevant journal

- We provide round the clock customer support

- Convenient online submission

- Thorough peer review

- Inclusion in PubMed and all major indexing services

- Maximum visibility for your research

Submit your manuscript at www.biomedcentral.com/submit 